\title{
Composite Fermions in Negative Effective Magnetic Field: A Monte-Carlo Study
}

\author{
Gunnar Möller ${ }^{a, b}$ and Steven H. Simon ${ }^{a}$ \\ ${ }^{a}$ Bell Laboratories, Lucent Technologies, Murray Hill, New Jersey 07974 \\ ${ }^{b}$ Laboratoire de Physique Théorique et Modèles Statistiques, 91406 Orsay, France
}

(Dated: February 21, 2005)

\begin{abstract}
The method of Jain and Kamilla [PRB 55, R4895 (1997)] allows numerical generation of composite fermion trial wavefunctions for large numbers of electrons in high magnetic fields at filling fractions of the form $\nu=p /(2 m p+1)$ with $m$ and $p$ positive integers. In the current paper we generalize this method to the case where the composite fermions are in an effective (mean) field with opposite sign from the actual physical field, i.e. when $p$ is negative. We examine both the ground state energies and the low energy neutral excitation spectra of these states. Using particle-hole symmetry we can confirm the correctness of our method by comparing results for the series $m=1$ with $p>0$ (previously calculated by others) to our results for the conjugate series $m=1$ with $p<0$. Finally, we present similar results for ground state energies and low energy neutral excitations for the states with $m=2$ and $p<0$ which were not previously addressable, comparing our results to the $m=1$ case and the $p>0, m=2$ cases.
\end{abstract}

\section{INTRODUCTION}

The composite fermion approach ${ }^{1}$ has had a great number of extremely impressive successes in describing the physics of electrons in high magnetic fields. In this picture, fractional quantum Hall systems in total magnetic field $B$ are described in terms of noninteracting "composite fermions" in an effective magnetic field $B_{\text {eff }}=B-2 m \phi_{0} n$, where $\phi_{0}=h c / e$ is the flux quantum, $n$ is the electron density, and $m$ is a positive integer. This maps, for example, fractional quantum Hall states at filling fractions of the form $\nu=n \phi_{0} / B=p /(2 m p+1)$ to integer quantum Hall states for the composite fermions at filling fraction $\nu_{\text {eff }}=n \phi_{0} /\left|B_{\text {eff }}\right|=|p|$. We will denote such composite fermions with $2 m$ flux quanta attached to them as ${ }^{2 m} \mathrm{CF}$.

Jain's original approach to composite fermions ${ }^{2}$ constructed highly accurate trial wavefunctions by taking simple wavefunctions for the noninteracting (composite) fermions in the effective magnetic field, multiplying by Jastrow factors, and then projecting the result into the lowest Landau level. The early successes of this method were impressive ${ }^{2}$, despite the fact that the method was limited by the extreme numerical difficulty of performing projections for systems with more than roughly 10 electrons.

A major theoretical breakthrough came when Jain and Kamilla ${ }^{3}$ discovered a new way of writing composite fermion trial wavefunctions (described below), which involves a very minor modification of the projection. These new trial states seemed to be just as good as the originally proposed wavefunctions and could be numerically generated even for systems with many electrons (40 electrons or more). Since that time, many important studies have been achieved using this method ${ }^{4-7}$. However, so far this method has been restricted to cases where the effective magnetic field has the same sign as the external magnetic field. Results using this method have been published for filling fractions of the form $\nu=p /(2 m p+1)$ with $p>0$ but not for $p<0$. In the current paper, we extend the work of Jain and Kamilla ${ }^{3}$ so that we are able to handle states with $p<0$. The $p<0$ states take more computational resources than the case of $p>0$, and the difference in the computational resources between the two cases increases with the absolute value of the effective flux. However, the computational problems turn out to be more severe for the smallest $|p|$, where the number of particles in the system increases most slowly with each flux added. Fortunately, we probably need not go to particularly large systems to understand the physics of small $|p|$. For large $|p|$, describing the approach to the Fermi liquid-like composite fermion state, the system size is already large for relatively small effective flux, i.e. the differences of the computational requirements in the case of $p>0$ and $p<0$ become relatively less important.

Although the case of negative $p$ has not previously been studied for large systems, we point out that for $m=$ 1 the series of states with negative $p$ and the series of states with positive $p$ are essentially equivalent due to an exact particle-hole symmetry in the lowest Landau level. In fact, below, we exploit this symmetry to check the validity of our method. Once we have verified the method, we can study the properties of the $m=2$ series for negative $p$ and compare the results to those of the positive $p$ members of this same series as well as to those of the $m=1$ series.

The outline of this paper is as follows. In section II we briefly review the Jain-Kamilla method. As mentioned above, the method has only been used previously for the case of positive $p$. In appendix A we show in detail how the crucial projection scheme of Jain-Kamilla can easily be generalized to handle negative $p$ also. It is easy to see from the result how much additional numerical complexity is involved for negative $p$. In section III we test our approach by examining the ${ }^{2} \mathrm{CF}$ series $\nu=p /(2 p+1)$. In particular, we examine ground state energies, excitation spectra, and energy gaps. We pay particular attention to the mass of the composite fermion and the scaling of the gap with $p$. For $p>0$, results are already available in the literature ${ }^{3-7}$. For $p<0$ we use our generalization of 
the Jain-Kamilla method to calculate energies directly, and we compare these energies to energies obtained by particle-hole conjugating the $p>0$ series. Appendix B describes the particle-hole conjugation transformation in depth. This comparison establishes the accuracy of our method. In section IV we move on to examine the ${ }^{4} \mathrm{CF}$ series of states $\nu=p /(4 p+1)$. Again, for $p>0$ some results are already available in the literature ${ }^{3-7}$. However, for $p<0$ our results are new. Again, we examine ground state energies, excitation spectra, energy gaps, and composite fermion effective masses. (By using particle-hole conjugation, we could give results for filling fractions $\nu=1-p /(4 p+1))$ similarly). We are able to make some comparison of our energy gaps to the experimental work of Pan et al. ${ }^{8}$.

Throughout this paper we assume complete spinpolarization of the electrons. This should be a reasonable assumption for real experiments at sufficiently high magnetic fields.

\section{THE JAIN-KAMILLA METHOD}

Jain's original proposal ${ }^{2}$ was to construct trial wavefunctions for fractional quantum Hall states by writing

$$
\Psi_{\text {Jain }}=\mathcal{P}\left\{\operatorname{det}\left[\psi_{i}\left(\vec{r}_{j}\right)\right] \Phi_{0}^{2 m}\right\},
$$

where the determinant is a Slater determinant of noninteracting single fermion wavefunctions $\psi_{i}$ in effective magnetic field $B_{\text {eff }}=B-2 m n \phi_{0}$, and $\mathcal{P}$ indicates projection to the lowest Landau level. Here, $\Phi_{0}$ is the wavefunction of a completely filled Landau level

$$
\Phi_{0}=\prod_{i<j}\left(z_{i}-z_{j}\right),
$$

where $z_{j}=\left(x_{j}+i y_{j}\right) / \ell_{0}$ is the dimensionless complex coordinate on the plane, $\ell_{0}=\sqrt{\hbar c / e B}$ and the usual Gaussian factors $\exp \left(-\frac{1}{4} \sum\left|z_{i}\right|^{2}\right)$ are understood to be included in the measure of the Hilbert space and will not be written explicitly for simplicity of notation.

Choosing a set of single particle wavefunctions $\psi_{i}$ to fill the $p$ lowest effective Landau levels (i.e., such that $\operatorname{det}\left[\psi_{i}\left(\vec{r}_{j}\right)\right]$ represents the ground state of an integer quantum Hall state $\nu=p$ ), one obtains through Eq. 1 extremely good trial wavefunctions for fractional quantum Hall states $\nu=p /(2 m p+1)$. As discussed above, the projection in Eq. 1 is exceedingly hard to implement for systems with more than roughly 10 electrons. For this reason, Jain and Kamilla ${ }^{3}$ looked for an essentially equivalent formulation that would be computationally simpler. In their approach they begin by rewriting the wavefunction as

$$
\Psi_{\text {Jain }}=\mathcal{P}\left\{\operatorname{det}\left[\psi_{i}\left(\vec{r}_{j}\right) J_{j}^{m}\right]\right\}
$$

where

$$
J_{j}=\prod_{k \neq j}\left(z_{k}-z_{j}\right)
$$

and then make the approximation that one can interchange the order of projection and taking the determinant to obtain a new trial wavefunction

$$
\Psi_{\mathrm{JK}}=\operatorname{det}\left[\tilde{\psi}_{i}\left(\vec{r}_{j}\right)\right] \Phi_{0}^{2 m}
$$

with

$$
\tilde{\psi}_{i}\left(\vec{r}_{j}\right)=J_{j}^{-m} \mathcal{P}\left\{\psi_{i}\left(\vec{r}_{j}\right) J_{j}^{m}\right\}
$$

Although $\Psi_{\text {JK }}$ appears to be a single Slater determinant, it is somewhat more complicated because each $\tilde{\psi}_{i}\left(\vec{r}_{j}\right)$ is actually a function of all of the particle positions through $J_{j}$. Nonetheless, this new trial wavefunction is far simpler to evaluate numerically. Furthermore, extensive numerical work ${ }^{3-7}$ has shown that for small systems $\Psi_{\mathrm{JK}}$ is just as good a trial state as $\Psi_{\text {Jain }}$ and that both are extremely accurate ${ }^{16}$. In the original work by Jain and Kamilla, it was shown how to calculate $\tilde{\psi}$ on a sphere for the case when $B_{\text {eff }}$ has the same sign as the magnetic field $B$ (i.e., $p>0$ ). In Appendix A we repeat the derivation for the case where $B_{\text {eff }}$ has the opposite sign from $B$ (i.e., $p<0$ ). A discussion is also given there of the relative computational effort required to perform the relevant computations numerically.

This technique allows one to also obtain low energy spectra of these fractional quantum Hall states, by similarly composite-fermionizing low energy excited states of noninteracting fermions as discussed in Ref. 4.

In this paper we will perform all calculations using a spherical geometry ${ }^{9}$ with a monopole of charge $N_{\phi}$ flux quanta at the center. The composite fermions then see an effective flux $2 q=N_{\phi}^{\text {eff }}=N_{\phi}-2 m(N-1)$. In the presence of this effective flux, single particle states are described by two quantum numbers, $l$ and $m$. Here $l=$ $|q|+n$ is the angular momentum with $n=0,1,2, \ldots$ corresponding to the "Landau level number" or "shell" index, and $m$ is the $z$-component of angular momentum. A state with $p$ filled composite fermion Landau levels corresponds to $n=|p|-1$.

A low energy exciton is now formed by taking a composite fermion out of the highest occupied shell (or Landau level) $l=l_{F}=|q|+|p|-1$ with some $m_{h}$ and putting it in the lowest unoccupied $l=l_{F}+1$ shell with some $m_{e}$. Choosing to work with states of zero total $z$-angular momentum, we take the state with $m_{e}=-m_{h}$ and write this state as $\left|m_{e}\right\rangle$. Using vector coupling (Clebsh-Gordon) coefficients ${ }^{12}$ we can construct exciton eigenstates of angular momentum $L$ as $^{4}$

$$
\Xi_{L}^{\text {exciton }}=\sum_{m_{e}=-l_{F}}^{l_{F}}\left|m_{e}\right\rangle\left\langle l_{F},-m_{e} ; l_{F}+1, m_{e} \mid L, 0\right\rangle,
$$

which serve as extremely accurate trial wavefunctions for the low energy excited states of the above discussed composite fermion ground states. 


\begin{tabular}{|c|c|c|c|c|c|c|c|c|c|c|c|c|}
\hline & \multicolumn{6}{|c|}{ Negative $p$ Trial Wavefunction } & \multicolumn{6}{|c|}{ Positive $p$ Trial Wavefunction } \\
\hline$N_{\phi}$ & $p$ & $N_{\phi}^{\text {eff }}$ & $N$ & $E_{g}$ & MR-gap & large k gap & $p$ & $N_{\phi}^{\mathrm{eff}}$ & $N$ & $E_{\bar{g}}:$ P-H conj & MR-gap & large k gap \\
\hline 9 & -2 & -1 & 6 & $-0.5391(1)$ & $0.0906(9)$ & $0.118(1)$ & 1 & 3 & 4 & $-0.53949(2)$ & $0.0928(2)$ & $0.118(2)$ \\
\hline 12 & & -2 & 8 & $-0.5338(1)$ & $0.091(1)$ & $0.115(1)$ & & 4 & 5 & $-0.53412(2)$ & $0.0931(2)$ & $0.1124(4)$ \\
\hline 15 & & -3 & 10 & $-0.5303(1)$ & $0.083(2)$ & $0.109(2)$ & & 5 & 6 & $-0.53090(2)$ & $0.0838(3)$ & $0.1079(4)$ \\
\hline 18 & & -4 & 12 & $-0.5282(1)$ & $0.080(2)$ & $0.104(3)$ & & 6 & 7 & $-0.52873(2)$ & $0.0801(4)$ & $0.1040(5)$ \\
\hline 21 & & -5 & 14 & $-0.5266(1)$ & $0.078(2)$ & $0.101(2)$ & & 7 & 8 & $-0.52721(2)$ & $0.0824(4)$ & $0.1021(4)$ \\
\hline 24 & & -6 & 16 & $-0.5257(1)$ & $0.079(5)$ & $0.100(3)$ & & 8 & 9 & $-0.52607(2)$ & $0.0768(5)$ & $0.1013(6)$ \\
\hline$\infty$ & & $-\infty$ & $\infty$ & $-0.5173(1)$ & $0.070(3)$ & $0.089(3)$ & & $\infty$ & $\infty$ & $-0.51803(3)$ & $0.069(4)$ & $0.0907(9)$ \\
\hline 21 & -3 & -1 & 12 & $-0.4985(1)$ & $0.061(2)$ & $0.067(2)$ & 2 & 3 & 10 & $-0.49870(5)$ & $0.061(1)$ & $0.069(1)$ \\
\hline 26 & & -2 & 15 & $-0.4980(1)$ & $0.056(3)$ & $0.063(3)$ & & 4 & 12 & $-0.49826(5)$ & $0.054(1)$ & $0.066(1)$ \\
\hline 31 & & -3 & 18 & $-0.4979(1)$ & $0.061(3)$ & $0.067(3)$ & & 5 & 14 & $-0.49803(5)$ & $0.053(1)$ & $0.063(1)$ \\
\hline 36 & & -4 & 21 & $-0.4979(1)$ & $0.056(3)$ & $0.069(3)$ & & 6 & 16 & $-0.49804(5)$ & $0.053(1)$ & $0.065(1)$ \\
\hline 41 & & -5 & 24 & $-0.4976(1)$ & $0.048(4)$ & $0.058(4)$ & & 7 & 18 & $-0.49797(6)$ & $0.050(2)$ & $0.064(2)$ \\
\hline$\infty$ & & $-\infty$ & $\infty$ & $-0.4968(2)$ & $0.042(8)$ & $0.060(8)$ & & $\infty$ & $\infty$ & $-0.4972(2)$ & $0.041(3)$ & $0.058(2)$ \\
\hline 37 & -4 & $\overline{-1}$ & 20 & $-0.4847(1)$ & $0.052(3)$ & $0.052(3)$ & 3 & 3 & $\overline{18}$ & $-0.48467(5)$ & $0.051(1)$ & $0.053(2)$ \\
\hline 44 & & -2 & 24 & $-0.4851(1)$ & $0.050(3)$ & $0.050(3)$ & & 4 & 21 & $-0.48525(7)$ & $0.041(2)$ & $0.052(2)$ \\
\hline 51 & & -3 & 28 & $-0.4855(1)$ & $0.042(4)$ & $0.048(4)$ & & 5 & 24 & $-0.48564(8)$ & $0.041(3)$ & $0.048(3)$ \\
\hline 58 & & -4 & 32 & $-0.4857(1)$ & $0.036(4)$ & $0.043(4)$ & & 6 & 27 & $-0.48593(6)$ & $0.041(3)$ & $0.046(3)$ \\
\hline$\infty$ & & $-\infty$ & $\infty$ & $-0.4875(1)$ & $0.014(7)$ & $0.030(6)$ & & $\infty$ & $\infty$ & $-0.48802(4)$ & $0.022(8)$ & $0.036(3)$ \\
\hline
\end{tabular}

TABLE I: Numerical results for energies and gaps at filling fractions $\nu=p /(2 p+1)$, given in units of $e^{2} / \epsilon \ell_{0}$. Calculations were performed using Monte-Carlo for ${ }^{2} \mathrm{CF}$ trial wavefunctions described in the text using $10^{7}$ samples. Particle-hole conjugate pairs should give precisely the same excitation energies (and the same ground state energies once Eq. 8 is used, as it is here, see below). In other words, if our results were exact, the right hand columns with positive $p$ should precisely match the left hand columns with negative $p$. Here, since we have used trial wavefunctions which are approximate (albeit extremely good), the agreement is not quite perfect, but it is extremely close. We note that the energies obtained by using the positive $p$ states are slightly lower, which means that the trial wavefunctions with positive $p$ (positive flux seen by the composite fermions) yield slightly better trial states. The number of electrons $N$ for particle-hole conjugate pairs sums to $N_{\phi}+1$, which is one filled Landau level. In this table, the ground state energy $E_{g}$ is presented for the negative $p$ case. For the positive $p$ case, in the column labeled " $E_{\bar{g}}$ : P-H conj" we have put the calculated ground state energy into Eq. 8 and presented the result for comparison with the corresponding negative $p$ states. The mageto-roton gap (MR-gap) is defined to be the lowest energy neutral excitation. The large $k$ gap is the gap measured at the highest possible angular momentum $L$ that we can construct using Eq. 7 which is $L_{\max }=2 l_{F}+1$. All values indicated for the thermodynamic limit have been extrapolated by a simple linear regression over the inverse particle number using this set of data only.

\section{PARTICLE-HOLE SYMMETRY AND RESULTS FOR $\nu=p /(2 p+1)$}

Using particle-hole symmetry of the lowest Landau level, one can exactly map states at filling fraction $\nu$ into states at filling fraction $1-\nu$ (so long as we maintain complete spin polarization). As mentioned above, in this paper we perform all calculations using a spherical geometry ${ }^{9}$. On the sphere, the lowest Landau level has $N_{\phi}+1$ single particle eigenstates with $N_{\phi}$ the total number of flux quanta penetrating the sphere. Thus states with $N$ electrons can be precisely mapped to their particle-hole conjugate states with $N_{\phi}+1-N$ electrons (i.e. with $N$ holes). In Appendix B we show that, on a sphere, given an eigenstate $\Psi$ with $N$ electrons and energy $E_{\Psi}$ one can write the energy $E_{\bar{\Psi}}$ of its particle-hole conjugate wavefunction $\bar{\Psi}$ as

$$
E_{\bar{\Psi}}=\left(1-\frac{2 N}{N_{\phi}+1}\right) E_{\text {filled }}+E_{\Psi}
$$

where $E_{\text {filled }}$ is the energy of the completely filled Landau level. This, of course, implies that the excitation spectrum of any given state is precisely the same as the excitation spectrum of its particle-hole conjugate state.

We will now focus on ${ }^{2} \mathrm{CF}$ states of the form $\nu=$ $p /(2 p+1)$. The state with $p$ is particle-hole conjugate of the state with $p \rightarrow(-p-1)$. (For example, $\nu=1 / 3$ which is $p=1$ is conjugate of $2 / 3$ which is $p=-2$ ). Extensive numerical work has already been performed for positive $p$, calculating accurate ground state energies and energy gaps $^{3-7}$. Using Eq. 8 this means that we already know the ground state energies and energy gaps for negative $p$. Here, however, we calculate these quantities directly using our negative $p$ trial wavefunctions and compare to the particle hole conjugated results to establish the validity of our approach.

Table I summarizes the numerical results for the ground state energies of states in this series calculated using Monte-Carlo ${ }^{3-717}$.

The ground-state energies we obtain using negative $p$ trial wavefunctions show an outstanding agreement with the values obtained by particle hole conjugating positive $p$ trial wavefunctions. We observed slight differences on the fourth significant digit, that show that the trial state with composite fermions in positive effective flux is very slightly better than the one with negative effective flux 


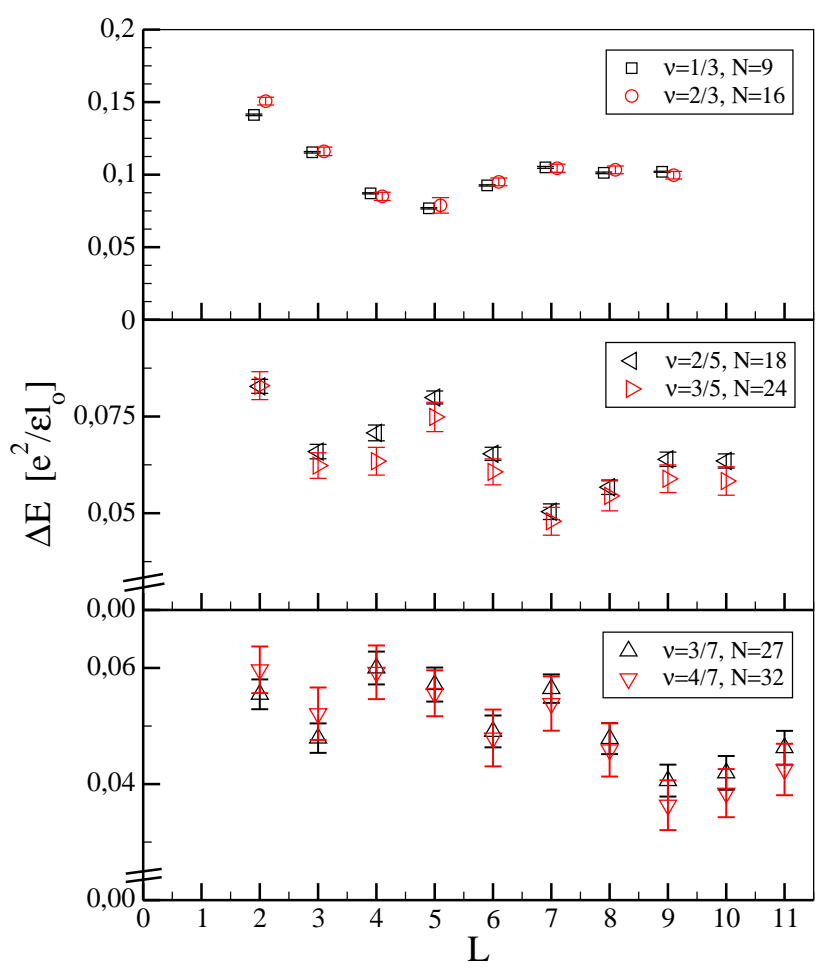

FIG. 1: (color online) Quantum Hall states that are particlehole conjugate of each other, are expected to have the same excitation spectrum. The low energy spectra shown here for for $\nu<\frac{1}{2}$ have been calculated with the method of Jain and Kamilla $^{4}$. The spectra for the particle-hole conjugate states with $\nu>\frac{1}{2}$ are calculated using the new negative effective flux trial wavefunctions described in this paper. If the calculations were exact (rather than just approximate) the corresponding spectra of the particle-hole conjugate pairs would match exactly. Although our calculations, being based on approximate trial wavefunctions for ${ }^{2} \mathrm{CFs}$, are not exact, we still see remarkably good agreement, suggesting that our new trial wavefunctions are approximately as accurate as the previously described trial wavefunctions for $\nu<\frac{1}{2}$. The spectra shown here correspond (as marked) to particle hole conjugate pairs at filling fractions, from top to bottom $1 / 3 \leftrightarrow 2 / 3,2 / 5 \leftrightarrow 3 / 5$ and $3 / 7 \leftrightarrow 4 / 7$ corresponding to flux 24,41 and $58 \phi_{0}$ respectively. Note that our new trial wavefunctions show an excellent reproduction of the increasingly nontrivial features as $\nu=1 / 2$ is approached. In the top panel the data points have been shifted slightly horizontally from integer angular momentum values for better distinguishability.

introduced here.

The excellent agreement of our negative $p$ wavefunctions with particle-hole conjugation of positve $p$ wavefunctions extends to the excited states, generated as outlined in section II. As examples, Figure 1 shows excitation spectra for $\nu=1 / 3,2 / 5$ and $3 / 7$ and their respective particle-hole conjugate states. We also give, in Table I values for magnetoroton gaps (which are the lowest energy neutral excitations) as well as large $k$ gaps (which are presumably the transport gap). These results, along with the excellent results for the ground-state energies confirm the validity of our approach to calculating CF wavefunctions at negative effective flux, which enables us to consider in the following section filling fractions above $1 / 4$ in the series $\nu=p /(4 p+1)$, that were previously inaccessible.

\section{RESULTS FOR $\nu=p /(4 p+1)$}

The ${ }^{4} \mathrm{CF}$ series of composite fermion states, corresponding to filling fractions around $\nu=1 / 4$, has been the subject of some recent experimental work ${ }^{8}$, yet the branch of filling fractions above $1 / 4$ has been mostly inaccessible to numerical investigations. The numerical approach we took for examining these states permits us to calculate the excitation spectra for systems with a moderate number of effective magnetic flux quanta $N_{\phi}^{\text {eff }}=2 q$ for the CF-system. For negative $p$, the calculational complexity of the wavefunction increases with $q$, consequently the achievable system size is reduced compared to the systems with positive $p$. For this very reason, extrapolation of the results to infinite system size in order to obtain the gap in the thermodynamic limit, and thus the mass of the CF, is difficult for these states. Nonetheless, interesting observations can be made already from the finite size data. The excitation spectra associated with $p$ and $-p$ show a striking similarity in structure at the same system size (see Fig. 2). These similarities, though apparent already for $|p|=2$ become even more clear as $|p|$ increases and $\nu=1 / 4$ is approached. Note that same values for the relative angular momentum $L$ translate into different absolute wave vectors of the corresponding excited state for different filling factors, since $k \propto L / \sqrt{N_{\phi}}$.

Table II summarizes the numerical results for the groundstate energies and gaps of states around $\nu=1 / 4$, and Figure 2 shows several examples of excitation spectra revealing the above mentioned similarity.

It is interesting to note that the $p \leftrightarrow-p$ similarity in spectra found here differs from the particle-hole symmetry correspondence of $p$ with the $-p-1$ states in the $\nu=p /(2 p+1)$ series shown above in section III. One might naively expect that the particle-hole symmetry $p \leftrightarrow-p-1$ for states in the ${ }^{2} \mathrm{CF}$ series $\nu=p /(2 p+1)$ above survives the composite fermionization attachment of two more Jastrow factors and results in an approximate symmetry for $\nu=p /(4 p+1)$ states. For example, we could take the symmetry related $m=1$ pair $(p=-3, N=12)$ and $(p=2, N=10)$ at $N_{\phi}=21$ on Table I and add two Jastrow factors so $N_{\phi} \rightarrow N_{\phi}+2(N-1)$ resulting in an approximate symmetry relating $\left(p=-3, N=12, N_{\phi}=43\right)$ to $\left(p=2, N=10, N_{\phi}=39\right)$ in Table II. However, when we examine the overall shape of the resulting excitation spectra, we find very little relation between the two spectra related by $p \leftrightarrow-p-1$ for ${ }^{4}$ CFs. In contrast we see in Figure 2 that the $p \leftrightarrow-p$ related states have quite similar dispersions, and the similarity appears to increase with increasing $|p|$. 


\begin{tabular}{|c|c|c|c|c|c|c|c|c|c|c|c|c|}
\hline & \multicolumn{6}{|c|}{ Negative $p$ Trial Wavefunction } & \multicolumn{6}{|c|}{ Positive $p$ Trial Wavefunction } \\
\hline$N$ & $p$ & $N_{\phi}^{\mathrm{eff}}$ & $N_{\phi}$ & $E_{g}$ & MR-gap & large $k$ gap & $p$ & $N_{\phi}^{\text {eff }}$ & $N_{\phi}$ & $E_{g}$ & MR-gap & large $k$ gap \\
\hline 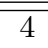 & $\mid-1$ & 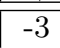 & 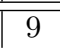 & $\overline{-0.47481(3)}$ & $\overline{0.0929(3)}$ & $\overline{0.1241(3)}$ & 1 & $\overline{3}$ & 15 & $\overline{-0.37706(1)}$ & $\overline{0.0169(1)}$ & $\overline{0.0305(1)}$ \\
\hline 5 & & -4 & 12 & $-0.45940(3)$ & $0.0939(4)$ & $0.1224(4)$ & & 4 & 20 & $-0.36501(1)$ & $0.0203(1)$ & $0.0276(1)$ \\
\hline 6 & & -5 & 15 & $-0.44996(3)$ & $0.0837(4)$ & $0.1183(4)$ & & 5 & 25 & $-0.35800(1)$ & $0.0132(1)$ & $0.0251(1)$ \\
\hline 7 & & -6 & 18 & $-0.44355(3)$ & $0.0812(3)$ & $0.1135(4)$ & & 6 & 30 & $-0.35303(1)$ & $0.0147(2)$ & $0.0241(2)$ \\
\hline 8 & & -7 & 21 & $-0.43895(3)$ & $0.0830(4)$ & $0.1110(5)$ & & 7 & 35 & $-0.34955(1)$ & $0.0151(2)$ & $0.0243(2)$ \\
\hline 9 & & -8 & 24 & $-0.43540(4)$ & $0.0781(5)$ & $0.1083(5)$ & & 8 & 40 & $-0.34686(2)$ & $0.0124(3)$ & $0.0245(3)$ \\
\hline 20 & & - & - & - & - & - & & 19 & 95 & $-0.33577(2)$ & $0.0109(5)$ & $0.0242(5)$ \\
\hline$-\infty$ & & $\infty$ & $\infty$ & $-0.40999(4)$ & $0.0668(5)$ & $0.097(3)$ & & $\infty$ & $\infty$ & $-0.32748(8)$ & $0.009(2)$ & $0.021(1)$ \\
\hline 6 & $\overline{-2}$ & -1 & 19 & $-0.40432(3)$ & $0.0243(2)$ & $0.0243(2)$ & 2 & 1 & 21 & $-0.38699(1)$ & $0.0182(2)$ & $0.0182(2)$ \\
\hline 8 & & -2 & 26 & $-0.39826(3)$ & $0.0231(3)$ & $0.0257(3)$ & & 2 & 30 & $-0.37411(1)$ & $0.0186(2)$ & $0.0193(2)$ \\
\hline 10 & & -3 & 33 & $-0.39484(3)$ & $0.0190(3)$ & $0.0283(3)$ & & 3 & 39 & $-0.36726(1)$ & $0.0142(4)$ & $0.0220(3)$ \\
\hline 12 & & -4 & 40 & $-0.39257(3)$ & $0.0207(4)$ & $0.0288(4)$ & & 4 & 48 & $-0.36276(2)$ & $0.0128(3)$ & $0.0219(4)$ \\
\hline 14 & & -5 & 47 & $-0.39092(3)$ & $0.0179(5)$ & $0.0275(5)$ & & 5 & 57 & $-0.35969(2)$ & $0.0125(4)$ & $0.0208(5)$ \\
\hline 16 & & -6 & 54 & $-0.38976(3)$ & $0.0203(4)$ & $0.0268(5)$ & & 6 & 66 & $-0.35742(2)$ & $0.0129(5)$ & $0.0183(5)$ \\
\hline 18 & & -7 & 61 & $-0.38885(3)$ & $0.0176(8)$ & $0.0235(9)$ & & 7 & 75 & $-0.35569(2)$ & $0.0108(6)$ & $0.0179(4)$ \\
\hline$\infty$ & & $-\infty$ & $\infty$ & $-0.38163(3)$ & $0.0151(1)$ & $0.0276(2)$ & & $\infty$ & $\infty$ & $-0.34277(5)$ & $0.0077(13)$ & $0.020(2)$ \\
\hline$\overline{12}$ & $\overline{-3}$ & $\overline{-1}$ & $\overline{43}$ & $-0.38486(3)$ & $0.0152(5)$ & $0.0152(5)$ & 3 & $\overline{1}$ & 45 & $-0.37306(2)$ & $\overline{0.0132(4)}$ & $0.0132(4)$ \\
\hline 15 & & -2 & 54 & $-0.38266(3)$ & $0.0154(7)$ & $0.0169(7)$ & & 2 & 58 & $-0.36765(3)$ & $0.0137(6)$ & $0.0137(6)$ \\
\hline 18 & & -3 & 65 & $-0.38126(3)$ & $0.0140(8)$ & $0.0190(8)$ & & 3 & 71 & $-0.36427(3)$ & $0.0119(7)$ & $0.0171(6)$ \\
\hline 21 & & -4 & 76 & $-0.38017(3)$ & $0.0126(8)$ & $0.0192(10)$ & & 4 & 84 & $-0.36182(2)$ & $0.0120(8)$ & $0.0175(8)$ \\
\hline 24 & & -5 & 87 & $-0.37940(3)$ & $0.0126(10)$ & $0.0196(9)$ & & 5 & 97 & $-0.36006(4)$ & $0.012(1)$ & $0.017(1)$ \\
\hline 27 & & -6 & 98 & $-0.37883(3)$ & $0.0134(14)$ & $0.0195(14)$ & & 6 & 110 & $-0.35869(2)$ & $0.010(1)$ & $0.016(1)$ \\
\hline$\infty$ & & $-\infty$ & $\infty$ & $-0.37404(3)$ & $0.010(1)$ & $0.0238(8)$ & & $\infty$ & $\infty$ & $-0.34842(4)$ & $0.0088(12)$ & $0.021(2)$ \\
\hline 20 & -4 & -1 & 75 & $-0.37206(3)$ & $0.0123(6)$ & $0.0123(6)$ & 4 & 1 & 77 & $-0.36788(3)$ & $0.0109(7)$ & $0.0109(7)$ \\
\hline 24 & & -2 & 90 & $-0.37172(4)$ & $0.010(1)$ & $0.0117(10)$ & & 2 & 94 & $-0.36491(3)$ & $0.0118(9)$ & $0.013(1)$ \\
\hline 28 & & -3 & 105 & $-0.37165(4)$ & $0.010(1)$ & $0.0148(7)$ & & 3 & 111 & $-0.36285(3)$ & $0.011(1)$ & $0.012(1)$ \\
\hline 32 & & -4 & 120 & $-0.37142(3)$ & $0.010(1)$ & $0.013(1)$ & & 4 & 128 & $-0.36133(3)$ & $0.0093(9)$ & $0.014(1)$ \\
\hline 36 & & -5 & 135 & $-0.37138(2)$ & $0.012(1)$ & $0.016(1)$ & & 5 & 145 & $-0.36021(3)$ & $0.011(1)$ & $0.014(2)$ \\
\hline$\infty$ & & $-\infty$ & $\infty$ & $-0.3705(1)$ & $0.010(3)$ & $0.019(3)$ & & $\infty$ & $\infty$ & $-0.35129(5)$ & $0.0087(19)$ & $0.017(2)$ \\
\hline
\end{tabular}

TABLE II: Numerical results from Monte Carlo calculations of ${ }^{4} \mathrm{CF}$ wavefunctions for groundstate energies and gaps at filling fraction $\nu=p /(4 p+1)$, given in units of $e^{2} / \epsilon \ell_{0}$. Alongside the newly calculated negative $p$ Quantum Hall states of this series above $\nu=1 / 4$, we give the results for states with the respective positive $p$ and equal particle number for comparison. We find that the similarity between the results for $p$ and $-p$ increases with increasing $p$. Two excitation energies are given from the spectra of neutral excitations: the magneto-roton gap as the lowest lying excitation and the large $k$ gap measured at the highest possible angular momentum. Extrapolation to the thermodynamic limit has been performed using the above given data by a simple linear regression over the inverse particle number (see Figure 3). In the case of groundstate energies, this extrapolation is based on density corrected values ${ }^{13}\left(E_{g}^{\text {corr }}=E_{g} \sqrt{\nu N_{\phi} / N}\right)$.

The existence of this similarity is perhaps not completely unexpected. Within a mean-field version ChernSimons theory of composite fermions ${ }^{14}$ one would expect the gaps to have this $p \leftrightarrow-p$ symmetry as well as having a symmetry in the excitation spectrum. However, beyond mean field theory, there is no clear reason to expect the symmetry to be preserved except for at large $|p|$.

We comment that another interesting test of our approach can be obtained by constructing a trial wavefunction for $\nu=1 / 3$ as the $p=-1$ member of the $\nu=p /(4 p+1)$ series. We find that the ground state energy of this wavefunction is, within numerical precision, precisely the same as that of the Laughlin $\nu=1 / 3$ trial wavefunction, which leads us to believe that we have precisely constructed that state. Similarly, we can examine the excitation spectrum of $\nu=1 / 3$ by using a ${ }^{2} \mathrm{CF}$ wavefunction of the series $p /(2 p+1)$ with $p=1$ or by using a ${ }^{4} \mathrm{CF}$ wavefunction of the series $p /(4 p+1)$ with $p=-1$. We find that the spectra obtained in these two approaches are quite similar to each other (albeit not quite identical), which gives us still further confidence in our approach. The values of the magneto-roton gap appear to be almost exactly the same in both cases. Yet, examining the large $k$ gap, the ${ }^{4} \mathrm{CF}$ trial wavefunction using negative flux $(p=-1)$ yields a slightly larger value, which decreases slightly more rapidly with the system size though, and seems to extrapolate to almost the same value at infinite $N$ (see Figure 3 ).

Figure 3 shows the extrapolation to the thermodynamic limit of the data given in Table II, comparing gaps at $p$ with those of states at $-p$. A comparison of the excitations at $\nu=1 / 3$ considered in the two different manners discussed above is also displayed there. Further, this figure gives us an idea of the quality of our extrapolation. Where the extrapolation is not particularly smooth, we cannot claim to deliver more than a rough result. The 


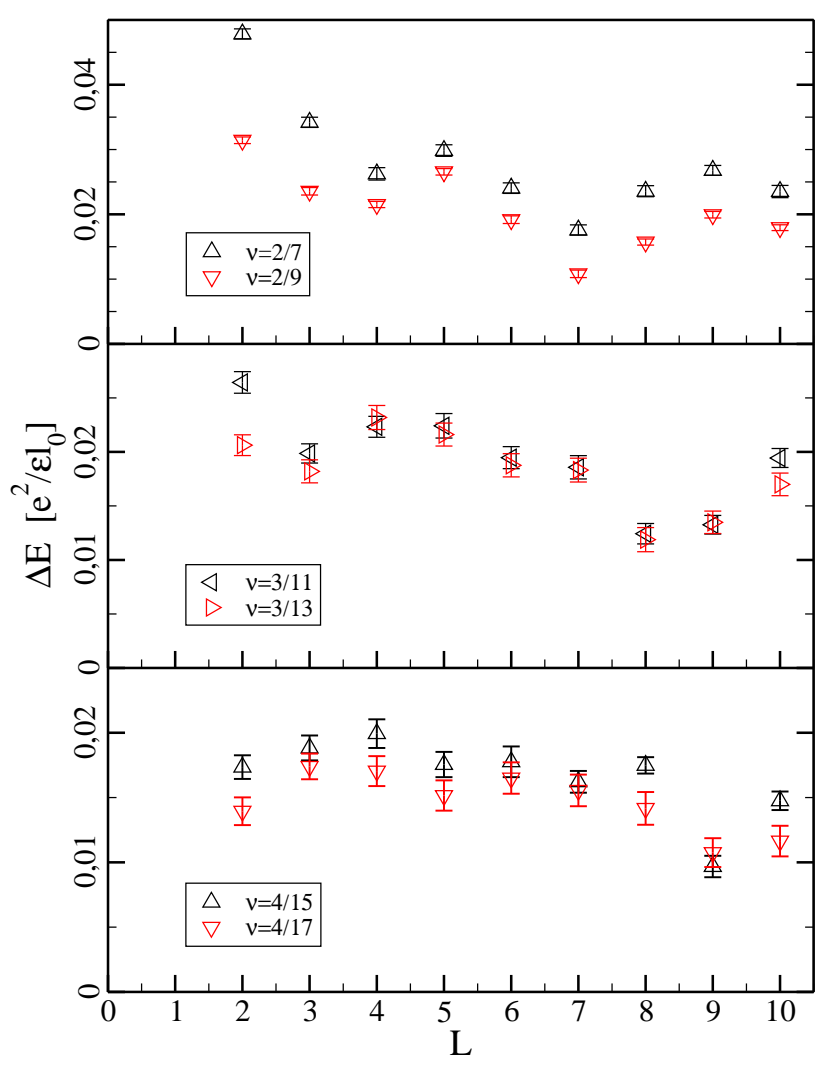

FIG. 2: (color online) The method developed here permits us to calculate the dispersion curves for the low energy excitations at filling fractions $p /(4 p+1)$ for negative $p$, i.e. above a quarter filling, that were previously inaccessible. A comparison to the spectra of states below and above $\nu=1 / 4$ shows, that the excitation spectra for $p$ and $-p$ show very similar features and seem to become more similar as $p$ is increased. As above, the $p>0$ spectra are calculated using the method of Jain and Kamilla ${ }^{4}$ whereas the $p<0$ spectra are calculated using the method discussed in the current paper.

extrapolation of $p= \pm 2$ appears to be the most difficult, since it is not easy to distinguish a clear linear dependence of the large $k$ gap as a function of $N^{-1}$ for the initial data set. The reason for this problem appears to be that the magnetoroton gap is located at a large value of $L$, so that in the smaller systems, the magnetoroton gap is located very close to the largest $k$ available, or even coincides with this point. For the $p=2$ case, we can see the error in this extrapolation clearly by comparing our extrapolated result to a result of a similar calculation using larger system sizes from Ref. 6. This comparison is show in Figure 4 (see below). We might guess that the error in extrapolation for $p=-2$ is of similar magnitude.

In order to obtain the composite fermion effective mass, we equate the activation gap $\Delta$ (determined from the excitation energy at the maximum angular momentum, i.e. biggest particle-hole separation) to the cyclotron

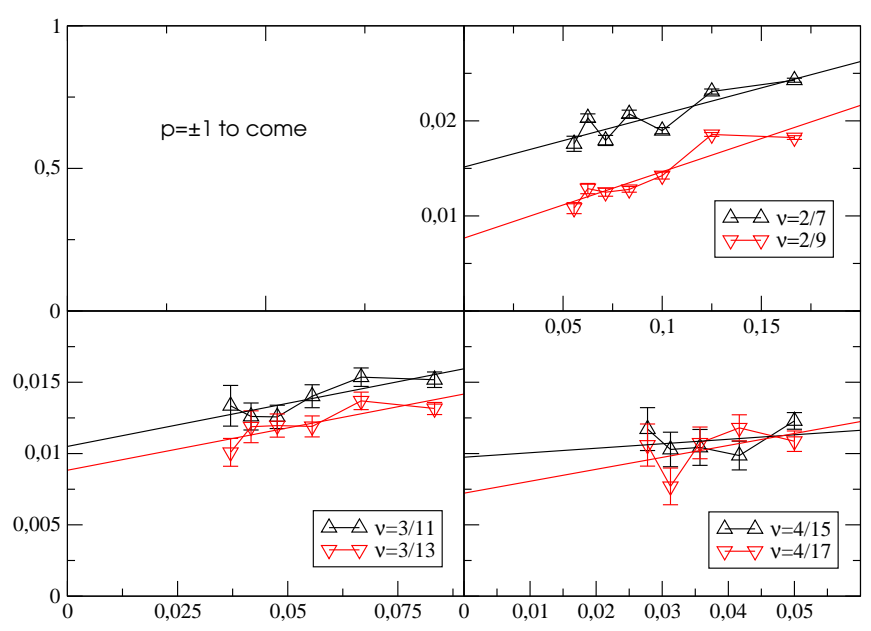

FIG. 3: (color online) This figure illustrates the extrapolation of the gaps to the thermodynamic limit for different filling fractions in the series $p /(4 p+1)$ by means of a simple linear regression of the available data points over the reciprocal particle number. States with $p$ and $-p$ are displayed together. The state at $\nu=1 / 3$ can be obtained in two different manners, i.e. as ${ }^{4} \mathrm{CF}$ in negative flux or as ${ }^{2} \mathrm{CF}$ in positive flux, which accounts for the two additional sets of data in the upper left. The magnetoroton gaps of these different $\nu=1 / 3$ states are close to indistinguishable, whereas the large $k$ gap is slightly bigger for negative effective flux. As discussed in the text, extrapolation is least certain for $p= \pm 2$ due to finite size effects.

energy of CFs in their effective magnetic field:

$$
\Delta_{\nu(m, p)}=\frac{\hbar e\left|B_{\mathrm{eff}}\right|}{m^{*} c}=\frac{\hbar^{2}}{|2 m p+1| m^{*} \ell_{0}^{2}} .
$$

Since the gap is measured in units of the Coulomb interaction, we write $\Delta=\left(e^{2} / \epsilon \ell_{0}\right) \delta$. Further taking into account $\epsilon_{r}=12.8$ for GaAs and the free electron mass as our point of reference, we find the dimensionless normalized ${ }^{18}$ effective mass $m_{\text {nor }}^{*}=m^{*} /\left(m_{e} \sqrt{B_{\nu}[T]}\right)$ to be given by $m_{\text {nor }}^{*}=0.0264 /(|2 m p+1| \delta)$. The ${ }^{4} \mathrm{CF}-$ masses we obtain are displayed in Figure 4 together with the large $k$ gaps from which they are deduced. In addition, for $\nu<1 / 4$ (positive $p$ ) we have shown data from Ref. 6 where larger systems were used than we have used here (When we also use larger system sizes, our results agree very will with those of Ref. 6 ). For $\nu=1 / 3$, as discussed above, we have shown results that treat this either as a $p=-1$ point of the $p /(4 p+1)$ series or a $p=1$ point of the $p /(2 p+1)$ series. For the latter case, we have likewise included the result from the above mentioned larger system calculation.

In Figure 4, we have intentionally displayed data extrapolated from a restricted set of small systems sizes with positive $p$ so as to match the same set of system sizes that we study for negative $p$ where we cannot go to very large systems. One can estimate the finite size error for our negative $p$ calculations by examining the deviations between these restricted extrapolations at positive 


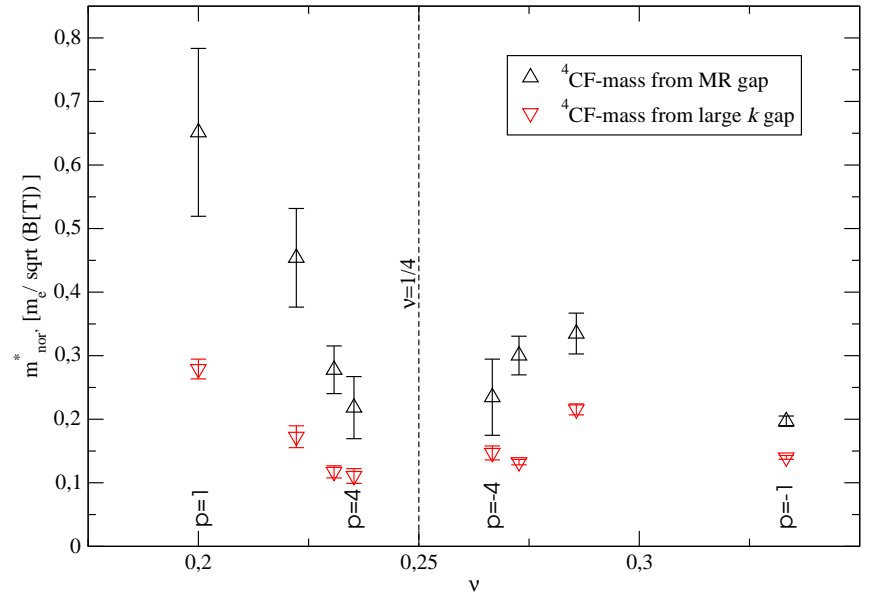

FIG. 4: (color online) Masses of the composite fermions near $\nu=1 / 4$ and extrapolated large $k$ gaps from which they were deduced at filling fractions of the series $\nu=p /(4 p+1)$. Data from Ref. 6 is from larger system size calculations and should be considered to be more accurate. Comparing these more accurate results to our smaller system calculations gives us an estimate of the finite size errors of our results which presumably will hold even in the $p<0$ case. Except for the marked asymmetry between $\nu=1 / 3$ and $\nu=1 / 5$ we do not see much sign of an asymmetry around $\nu=1 / 4$ which is observed experimentally ${ }^{8}$. We also do not see signs of the experimentally observed diverging effective mass as $\nu=1 / 4$ is approached. Our values of the effective mass are somewhat less than that obseved experimentally in general. However, it is known that finite well width corrections tend to increase the effective masses ${ }^{6}$. Two additional data points are shown in this figure (not mentioned in the legend) which give results obtained by treating $\nu=1 / 3$ as a ${ }^{2} \mathrm{CF}$ state.

$p$ compared to the larger system calculations of Ref. 6 (also displayed in our figure).

The behavior of the negative $p$ fractions seems to roughly mirror the behavior of the positive $p$ fractions, at least for $|p| \geq 2$. As was seen previously in Ref. 6 for $p>0$ it is seen that the effective mass increases with $\left|B_{\text {eff }}\right|$. As $\nu=1 / 4$ is approached from either side, it is not clear if the effective mass will converge to a constant as would be predicted by theory (up to logarithmic corrections $^{14}$ ). In the experiments of Ref. 8, a striking asymmetry of the effective mass beween the high field and low field sides of $\nu=1 / 4$ has been observed. While we cannot rule out some asymmetry from our data, we certainly cannot claim to see the extremely strong differences that are observed experimentally. This, however, is not surprising. Experimentally, the asymmetry is attributed to the proximity of a Wigner crystal state ${ }^{8}$. Since we are using a trial wavefunction approach, we should not see the effects of any imminent phase transition.

Perhaps the most interesting data point in Figure 4 is the one at $\nu=1 / 3$. Whether we treat this point as the $p=-1$ member of the series $p /(4 p+1)$ or the $p=1$ member of the series $p /(2 p+1)$, we find almost identical results of a very large gap, which establishes a continuity between the $\mathrm{CF}$ masses around $\nu=1 / 2$ and $\nu=1 / 4$. Furthermore, we note that this point is quite asymmetric with its reflection at $\nu=1 / 5$. Certainly the hypothesis of constant effective composite fermion mass does not extend all the way from $\nu=1 / 4$ out to both $\nu=1 / 5$ and $\nu=1 / 3$. Below quarter filling, one observes a continuous increase of this mass. A similar trend appears at small values of the effective magnetic field above this point, but then the mass drops down again at $\nu=1 / 3$, the final point of this series.

Generally, our values for the effective mass seem to be lower than those measured in the experiments of Ref. 8 by a factor of roughly 2.5. This error is rather expected, since similar discrepancies have been observed in previous studies based on the composite fermion picture ${ }^{6}$. It is known, however, that taking into account the finite width of the 2D electron gas changes the interaction so as to increase the effective mass ${ }^{6}$.

We note that we are not able to find any evidence of the divergence of $m^{*}$ as we approach $\nu=1 / 4$ from either side, which is observed experimentally in Ref. 8. This is not surprising for several reasons. First of all, the experiment only sees strong divergences extremely close to $\nu=1 / 4$ - which we cannot access numerically. Furthermore, one might suspect that the disorder might be the source of the divergences in the measurements used by Pan. More importantly, however, even if there were genuine infra-red divergences ${ }^{14}$ of the effective mass as $\nu=1 / 4$ is approached, one would not necessarily expect such divergences to be properly represented in a trial wavefunction approach.

We emphasize that the most important achievement of this paper is not any particular numerical result. If genuine numbers were desired for comparison to experiment, we would want to use a more realistic interaction, accounting for finite well width ${ }^{6}$, as well as perhaps Landau-level mixing, and we would want to use a more powerful computer to analyze ever larger systems. Instead we would like to emphasize in this paper that we have clearly demonstrated that we can extend the approach of Jain and Kamilla ${ }^{3}$ to treat negative effective magnetic field, and we can study these negative $p$ composite fermion wavefunctions for reasonably large systems, which has not been done before. In this paper we have tested this method by using particle-hole symmetry for the ${ }^{2} \mathrm{CF}$ series and we have found our method to be quite accurate. We have then applied this method to ${ }^{4} \mathrm{CFs}$ to study, for the first time, large systems for filling fractions $p /(4 p+1)$ with negative $p$. Our main physical result is that the effective mass appears to be roughly symmetric around and close to $\nu=1 / 4$ although larger system calculations would be desirable.

The authors acknowledge helpful conversations with E. H. Rezayi. G.M. acknowledges support from the French Ministry of Science, and thanks both the Ecole Doctorale de Paris and Lucent Technologies for their support that made participation in this research project possible. 


\section{Appendix A: CF wavefunctions with negative effective flux}

The starting point for the composite fermion trial wavefunctions $^{3}$ are the single particle eigenfunctions of the quantum mechanical problem of a particle in a magnetic monopole field on a sphere. (We use spherical coordinates with the azimuth $\theta$ ranging from 0 to $\pi$, and $\phi$ the longitude ranging from 0 to $2 \pi$ ). The monopole harmonics are given by ${ }^{10}$

$$
Y_{n, m}^{q}(\Omega)=2^{m} M_{q, n, m}(1-x)^{\alpha / 2}(1+x)^{\beta / 2} P_{g}^{\alpha, \beta}(x) e^{i m \phi}
$$

with $\alpha=-q-m, \beta=q-m, g=|q|+n+m, x=\cos \theta$,

$$
M_{q, n, m}=\sqrt{\frac{2|q|+2 n+1}{4 \pi} \frac{(|q|+n-m) !(|q|+n+m) !}{n !(2|q|+n) !}}
$$

and $P_{g}^{\alpha, \beta}(x)$ are the Jacobi polynomials. This monopole harmonic represents an eigenstate of a particle on a sphere in a radial magnetic field with $2 q$ flux quanta penetrating the sphere, where a positive sign refers to outwards pointing flux. Here, the angular momentum of the eigenstate is $l=|q|+n$ and the $z$ component of the angular momentum $m \in\{-l, \ldots, l\}$. Further, $n$ is the LL index $n=0,1, \ldots$. The above expression assumes the Haldane gauge ${ }^{9}$, where the singularities of the vector potential are chosen to be located symmetrically on both north- and southpole of the sphere. Here, we focus on the case where $q<0$, since the $q>0$ case has already been discussed in detail in Ref. 3. For the rest of this paragraph, we thus assume $q<0$. Expanding the Jacobi polynomials in terms of the spinor coordinates $u=\cos (\theta / 2) e^{-i \phi / 2}$ and $v=\sin (\theta / 2) e^{i \phi / 2}$, one obtains

$$
\begin{aligned}
& Y_{n, m}^{q<0}(\Omega)=(-1)^{n} M_{q, n, m}\left(u^{*}\right)^{-q+m}\left(v^{*}\right)^{-q-m} \times \\
& \sum_{s=0}^{n}(-1)^{s}\left(\begin{array}{c}
n \\
s
\end{array}\right)\left(\begin{array}{c}
2|q|+n \\
|q|+m+s
\end{array}\right)\left(u^{*} u\right)^{s}\left(v^{*} v\right)^{n-s} .
\end{aligned}
$$

This expression can equally well be obtained from the relation for complex conjugation of the monopole harmonics ${ }^{11}$, if one corrects Kamilla's formula by replacing $q$ by $|q|$ in the appropriate places. We now use the $Y_{n, m}^{q}$ as single particle wavefunctions (written as $\psi_{i}$ in the main text) and composite fermionize by attaching Jastrow factors. As discussed in the main text, we can construct the many particle composite fermion trial wavefunctions by bringing the Jastrow factors inside the Slater determinant of single particle states, which may then be projected individually. Note, that in the spherical geometry, the Jastrow factor becomes

$$
J_{j}=\prod_{k \neq j}\left(u_{k} v_{j}-u_{j} v_{k}\right)
$$

The details of the required projection $\mathcal{P}\left(Y_{n, m}^{q}\left(u_{i}, v_{i}\right) J_{i}^{p}\left(u_{1}, v_{1}, \ldots, u_{N}, v_{N}\right)\right) \quad$ are discussed next.
First, we remark that the Jastrow factor ${ }^{19} J_{i}^{p}$ is a LLL function, with $q^{\prime}=p(N-1)$ zeros in $u_{i}$, i.e. it is a LLL wavefunction for flux $q^{\prime}>0$. Since $N$ is in general a big number, we have $q^{\prime} \gg|q|$. The resulting wavefunction, in turn, has to be a valid wavefunction for a total number of flux $Q=q+q^{\prime}>0$. Secondly, since projection is a linear operation, we may consider the action of projection on each of the basis states $Y_{0, m^{\prime}}^{q^{\prime}}$ separately, by expanding $J_{i}^{p}$ in this basis. In general multiplication by a basis state $Y_{n, m}^{q}$ followed by projection can be described as a linear operator called hereafter $\mathfrak{Y}_{q, n, m}^{q^{\prime}}$.

$$
\mathcal{P} Y_{n, m}^{q} Y_{0, m^{\prime}}^{q^{\prime}}=\mathfrak{Y}_{q, n, m}^{q^{\prime}} Y_{0, m^{\prime}}^{q^{\prime}}
$$

Since we know the entire basis of the subspace that we project upon, namely the LLL for flux $Q$ with states $|M\rangle$, and $|M| \leq Q$, the projection operator is $\sum_{M}|M\rangle\langle M|$. We now show, how this leads to an expression for $\mathfrak{Y}_{q, n, m}^{q^{\prime}}$ as a differential operator in the coordinate representation, in which (A3) becomes

$$
\begin{array}{r}
\sum_{M=-Q}^{Q} Y_{0, M}^{Q}(\Omega) \int d \Omega^{\prime} Y_{0, M}^{Q *}\left(\Omega^{\prime}\right) Y_{n, m}^{q}\left(\Omega^{\prime}\right) Y_{0, m^{\prime}}^{q^{\prime}}\left(\Omega^{\prime}\right) \\
=\mathfrak{Y}_{q, n, m}^{q^{\prime}} Y_{0, m^{\prime}}^{q^{\prime}}(\Omega)
\end{array}
$$

Integration over the longitudinal angle $\phi$ singles out one nonzero scalar product for $M=m+m^{\prime}$, and the one remaining integral over the azimuthal angle $\theta$ yields a well known binomial coefficient. Simplifying the normalization factors of $Y_{0, m^{\prime}}^{q^{\prime}}$ on both sides, we have:

$$
\begin{aligned}
& (-1)^{n} M_{q, n, m} \sum_{s}(-1)^{s}\left(\begin{array}{c}
n \\
s
\end{array}\right)\left(\begin{array}{c}
2|q|+n \\
|q|+m+s
\end{array}\right) \\
\times & \left(N_{Q, 0, M}\right)^{2} 4 \pi \frac{\left(q^{\prime}-m^{\prime}+s\right) !\left(q^{\prime}+m^{\prime}+n-s\right) !}{\left(2 q^{\prime}+n+1\right) !} \\
\times & u^{q-m+q^{\prime}-m^{\prime}} v^{q+m+q^{\prime}+m^{\prime}}=\mathfrak{Y}_{q, n, m}^{q^{\prime}} u^{q^{\prime}-m^{\prime}} v^{q^{\prime}+m^{\prime}} .
\end{aligned}
$$

Using the explicit form of the normalization

$$
\left(N_{Q, 0, M}\right)^{2}=\frac{(2 Q+1) !}{4 \pi(Q+M) !(Q-M) !}
$$

and remarking that the fractions of factorials that are left in this expression equal those that appear by the multiple derivation of a monomial $u^{k}$

$$
\left(\frac{\partial}{\partial u}\right)^{s-q+m} u^{q^{\prime}+m^{\prime}+s}=\frac{\left(s+q^{\prime}+m^{\prime}\right) !}{(Q+M) !} u^{Q+M},
$$

we may deduce $\mathfrak{Y}_{q, n, m}^{q^{\prime}}$ by comparison of both sides:

$$
\begin{aligned}
\mathfrak{Y}_{q, n, m}^{q^{\prime}}= & \frac{(2 Q+1) !}{\left(2 q^{\prime}+n+1\right) !}(-1)^{n} M_{q, n, m} \times \\
& \sum_{s=0}^{n}(-1)^{s}\left(\begin{array}{c}
n \\
s
\end{array}\right)\left(\begin{array}{c}
2|q|+n \\
|q|+m+s
\end{array}\right) \times \\
& \left(\frac{\partial}{\partial u}\right)^{|q|+m+s} u^{s}\left(\frac{\partial}{\partial v}\right)^{|q|-m+n-s} v^{n-s} .
\end{aligned}
$$


Let us remark that this result reproduces the known result, that the projection on the LLL is achieved by performing the habitual procedure of moving all $u^{*}$ 's and $v^{*}$ 's to the far left, and replacing them with derivatives according to

$$
u^{*} \rightarrow \frac{\partial}{\partial u}, \text { and } \quad v^{*} \rightarrow \frac{\partial}{\partial v} .
$$

Nevertheless, performing this explicit projection gives us a supplementary information in the form of a weight factor $\frac{(2 Q+1) !}{\left(2 q^{\prime}+n+1\right) !}$ for the different Landau levels before projection, which of course does not matter for the problems discussed here, but may play a role in other $\operatorname{cases}^{15}$.

Practically, we would like to obtain a form of (A8) with the derivatives moved to the extreme right, which may be calculated using a straightforward application of the Leibniz rule for multiple derivatives in both $u$ and $v$ :

$$
\left(\frac{\partial}{\partial v}\right)^{\beta} v^{\gamma}=\sum_{\alpha=0}^{\beta} \frac{\beta !}{\alpha !}\left(\begin{array}{c}
\gamma \\
\beta-\alpha
\end{array}\right) v^{\gamma-\beta-\alpha}\left(\frac{\partial}{\partial v}\right)^{\alpha} .
$$

This yields a triple sum with the inner summation ranges being dependant on the outer summation index $s$. One finds that the summation ranges can be made independent of $s$ since the summand becomes zero outside of the given intervals. As such the sum over $s$ may be evaluated using

$$
\sum_{s=0}^{n}(-1)^{s}\left(\begin{array}{c}
n-\alpha-\alpha^{\prime} \\
s-\alpha
\end{array}\right)=(-1)^{\alpha} \delta_{n, \alpha+\alpha^{\prime}}
$$

Since the result yields a Kronecker delta, one of the remaining sums becomes trivial, and after shifting the remaining summation index, the final result is revealed to be exactly like (A8), but with all derivatives placed at the very right.

The projected composite fermion wavefunction is nothing but this operator applied to the single particle Jastrow factor:

$$
Y_{n, m}^{q}{ }^{C F}\left(\Omega_{i}\right)=\mathfrak{Y}_{q, n, m}^{q^{\prime}} J_{i}^{p} .
$$

In order to perform numerical calculations with this wavefunction, we need to evaluate the derivatives explicitly. One may use Jain and Kamilla's approach ${ }^{3}$, to commute the derivatives through the Jastrow factors as

$$
\left(\frac{\partial}{\partial u_{i}}\right)^{s}\left(\frac{\partial}{\partial v_{i}}\right)^{t} J_{i}^{p}=J_{i}^{p}\left[\hat{U}_{i}^{s} \hat{V}_{i}^{t} 1\right]
$$

with

$$
\hat{U}_{i}=J_{i}^{-p} \frac{\partial}{\partial u_{i}} J_{i}^{p} \text {, and } \hat{V}_{i}=J_{i}^{-p} \frac{\partial}{\partial v_{i}} J_{i}^{p}
$$

Constructing a many-particle wavefunction out of these projected CF wavefunctions in the form of a Slater determinant, one may factor out the Jastrow factors again, and thus obtains a form which resembles single particle wavefunctions, on a basis of projected states $\tilde{Y}_{n, m}^{q}$ with:

$$
\begin{aligned}
\tilde{Y}_{n, m}^{q}\left(\Omega_{i}\right)= & \frac{(2 Q+1) !}{\left(2 q^{\prime}+n+1\right) !}(-1)^{n} M_{q, n, m} \times \\
& \sum_{s=0}^{n}(-1)^{s}\left(\begin{array}{c}
n \\
s
\end{array}\right)\left(\begin{array}{c}
2|q|+n \\
|q|+m+s
\end{array}\right) \times \\
& u_{i}^{s} v_{i}^{n-s}\left[\hat{U}_{i}^{|q|+m+s} \hat{V}_{i}^{|q|-m+n-s} 1\right] .
\end{aligned}
$$

Of course, this only appears to be a one particle wavefunction, since there is an implicit dependence of the positions of all other electrons in the system hidden in the operators $\hat{U}_{i}$ and $\hat{V}_{i}$. The complexity of this expression increases with the total number of derivatives per term, given by $N_{\partial}^{q<0}=2|q|+n$ for negative $q$ compared to $N_{\partial}^{q>0}=n$ for positive effective flux.

\section{Appendix B: Particle-hole Conjugation}

In the lowest Landau level, on the sphere, there are $N_{\phi}+1$ single particle eigenstates where $N_{\phi}$ is the flux through the sphere. We label these eigenstates by the $z$-component of their angular momentum $m$.

For a two-body interaction we can write the Hamiltonian as

$$
H=\sum_{m_{1}, m_{2}, m_{3}, m_{4}} V_{m_{1}, m_{2}, m_{3}, m_{4}} c_{m_{1}}^{\dagger} c_{m_{2}}^{\dagger} c_{m_{3}} c_{m_{4}} .
$$

As usual, the normal ordering of the operators accounts for the uniform positive background. As usual, the fermion operators have anticommutation relations $\left\{c_{i}^{\dagger}, c_{j}\right\}=\delta_{i, j}$. The interaction matrix $V$ must have the following symmetries

$$
\begin{aligned}
V_{m_{1}, m_{2}, m_{3}, m_{4}} & =-V_{m_{2}, m_{1}, m_{3}, m_{4}}=-V_{m_{1}, m_{2}, m_{4}, m_{3}} \\
& =V_{m_{4}, m_{3}, m_{2}, m_{1}}^{*} .
\end{aligned}
$$

Furthermore, for any rotationally (translationally) invariant interaction, we must have angular momentum conservation, which implies that the matrix element is zero unless

$$
m_{1}+m_{2}=m_{3}+m_{4}
$$

We define the vacuum state $\left|0_{e}\right\rangle$, to be the state which contains no electrons at all. The filled Landau level, which we can also think of as the vacuum for holes is written as $\left|0_{h}\right\rangle=\mid$ Filled $\left._{e}\right\rangle=\prod_{m} c_{m}^{\dagger}\left|0_{e}\right\rangle$. It is convenient to introduce creation and annihilation operators $d, d^{\dagger}$ for holes, given by $d_{i}=c_{i}^{\dagger}, d_{i}^{\dagger}=c_{i}$ which also obey the usual anticommutations $\left\{d_{i}^{\dagger}, d_{j}\right\}=\delta_{i, j}$. We now rewrite the Hamiltonian in terms of these hole operators. Using the commutation relations as well as above described 
symmetries of $V$ we obtain

$$
\begin{aligned}
H & =\sum_{m_{1}, m_{2}, m_{3}, m_{4}} V_{m_{1}, m_{2}, m_{3}, m_{4}} d_{m_{1}} d_{m_{2}} d_{m_{3}}^{\dagger} d_{m_{4}}^{\dagger} \\
& =2 \sum_{m} U_{m}\left(1-2 d_{m}^{\dagger} d_{m}\right)+H_{d}
\end{aligned}
$$

where

$$
U_{m}=\sum_{m_{2}} V_{m, m_{2}, m_{2}, m}
$$

and

$$
H_{d}=\sum_{m_{1}, m_{2}, m_{3}, m_{4}} V_{m_{1}, m_{2}, m_{3}, m_{4}}^{*} d_{m_{1}}^{\dagger} d_{m_{2}}^{\dagger} d_{m_{3}} d_{m_{4}} .
$$

We show below that for any rotationally invariant interaction, $U_{m}$ is actually independent of $m$. Furthermore, it is very easy to show that the energy of the entirely filled Landau level is given by

$$
E_{\text {filled }}=2 \sum_{m_{1}, m_{2}} V_{m_{1}, m_{2}, m_{2}, m_{1}}=2 \sum_{m} U_{m}
$$

Thus we have

$$
H=\left(1-\frac{2 N_{h}}{N_{\phi}+1}\right) E_{\text {filled }}+H_{d}
$$

and $N_{h}$ is the number of holes (i.e., the eigenvalue of $\left.\sum_{m} d_{m}^{\dagger} d_{m}\right)$.
A general state containing $N$ electrons in the LLL can be written as:

$$
|\Psi\rangle=\sum_{\left\{m_{i}\right\}} a_{m_{1}, \ldots, m_{N}} c_{m_{1}}^{\dagger} \cdots c_{m_{N}}^{\dagger}\left|0_{e}\right\rangle
$$

To particle-hole conjugate this state we construct

$$
|\bar{\Psi}\rangle=\sum_{\left\{m_{i}\right\}} a_{m_{1}, \ldots, m_{N}}^{*} d_{m_{1}}^{\dagger} \cdots d_{m_{N}}^{\dagger}\left|0_{h}\right\rangle
$$

which is now a state containing $N$ holes, or $N_{\phi}+1-N$ electrons. Note that both of these states "live" in the same lowest Landau level which has $N_{\phi}+1$ single particle eigenstates (indexed by $m$ ).

Now, if $\Psi$ is an eigenstate of $H$ with eigenvalue $E_{\Psi}$ (assumed to be real), then, since $H_{d}$ has precisely the same structure as $H$ we see that $\bar{\Psi}$ is an eigenstate of $H_{d}$ with the same eigenvalue. Thus, we obtain the particle hole conjugation relation

$$
E_{\Psi}=\left(1-\frac{2 N_{h}}{N_{\phi}+1}\right) E_{\text {filled }}+E_{\bar{\Psi}}
$$

Lemma: $U_{m}$ is independent of $m$ :

If an interaction is rotationally invariant, we can choose any rotation $R$ and write

$$
V_{m_{1}, m_{2}, m_{3}, m_{4}}=\sum_{m_{1}^{\prime}, m_{2}^{\prime}, m_{3}^{\prime}, m_{4}^{\prime}} D_{m_{1}, m_{1}^{\prime}}^{l}(R) D_{m_{2}, m_{2}^{\prime}}^{l}(R) V_{m_{1}^{\prime}, m_{2}^{\prime}, m_{3}^{\prime}, m_{4}^{\prime}}\left[D_{m_{3}, m_{3}^{\prime}}^{l}(R)\right]^{*}\left[D_{m_{4}, m_{4}^{\prime}}^{l}(R)\right]^{*}
$$

where the $D$ 's are rotation matrices as in Ref. 12 and $l=2 N_{\phi}$. Setting $m_{2}=m_{3}$, and $m_{1}=m_{4}=m$ and summing over $m_{2}$ as prescribed in Eq. B5 we obtain

$$
U_{m}=\sum_{m_{1}^{\prime}, m_{2}^{\prime}} D_{m, m_{1}^{\prime}}^{l}(R) V_{m_{1}^{\prime}, m_{2}^{\prime}, m_{2}^{\prime}, m_{1}^{\prime}}\left[D_{m, m_{1}^{\prime}}^{l}(R)\right]^{*}
$$

where we have used the orthogonality ${ }^{12}$

$$
\sum_{m_{2}} D_{m_{2}, m_{2}^{\prime}}(R)\left[D_{m_{2}, m_{3}^{\prime}}(R)\right]^{*}=\delta_{m_{2}^{\prime}, m_{3}^{\prime}}
$$

as well Eq. B3. Since Eq. B13 must be true for any rotation, we can integrate over all rotations and use $\mathrm{u}^{12}$

$$
\int d R D_{m, m_{1}^{\prime}}^{l}(R)\left[D_{m, m_{1}^{\prime}}^{l}(R)\right]^{*}=\mathrm{constant}
$$

independent of $m$, which shows that $U_{m}$ is independent of $m$.
${ }^{1}$ For a review of Composite Fermions see "Composite Fermions", ed. O. Heinonen, World Scientific, 1998; and therein.

2 See J. K. Jain in "Perspectives in Quantum Hall Effects", ed. S. Das Sarma and A. Pinczuk, Wiley, 1997; and therein.
3 J. K. Jain and R. K. Kamilla, Phys. Rev. B 55, R4895R4898 (1997). Int. J. Mod. Phys. B11, 2621 (1997).

${ }^{4}$ R. K. Kamilla and J. K. Jain, Phys. Rev. B 55, R13417R13420 (1997).

${ }^{5}$ R. K. Kamilla, J. K. Jain, and S. M. Girvin, Phys. Rev. B 
56, 12411-12416 (1997); K. Park and J. K. Jain, Phys. Rev. Lett. 80, 4237-4240 (1998); K. Park, V. Melik-Alaverdian, N. E. Bonesteel, and J. K. Jain, Phys. Rev. B 58, R10167R10170 (1998); K. Park and J. K. Jain, Phys. Rev. Lett. 83, 5543-5546 (1999); V. W. Scarola, K. Park, and J. K. Jain, Phys. Rev. B 61, 13064-13072 (2000); K. Park and J. K. Jain, Phys. Rev. Lett. 84, 5576-5579 (2000); T. Sbeouelji, K. Park, J. K. Jain, and N. Meskini, Phys. Rev. B 62, R4802-R4804 (2000); K. Park and J. K. Jain, Phys. Rev. B 62, R13274-R13277 (2000); V. W. Scarola, K. Park, and J. K. Jain, Phys. Rev. B 62, R16259-R16262 (2000); S. S. Mandal and J. K. Jain, Phys. Rev. B 63, 201310 (2001); Phys. Rev. B 64, 081302 (2001); Phys. Rev. B 64, 125310 (2001); Phys. Rev. Lett. 89, 096801 (2002); T. Sbeouelji and N. Meskini, Phys. Rev. B 64193305 (2001).

${ }^{6}$ X. Zu, K. Park, and J. K. Jain, Phys. Rev. B 61, R7850R7853 (2000).

7 K. Park, N. Meskini, and J. K. Jain, J. Phys. Cond Matt 11, 7283 (1999); V. W. Scarola, S. Y. Lee, and J. K. Jain, Phys. Rev. B 66, 155320 (2002); K. Park and J. K. Jain, Phys. Rev. Lett. 81, 4200-4203 (1998).

${ }^{8}$ W. Pan, H. L. Stormer, D. C. Tsui, L. N. Pfeiffer, K. W. Baldwin, and K. W. West, Phys. Rev. B 61, R5101-R5104 (2000).

9 F. D. M. Haldane, Phys. Rev. Lett. 51, 605 (1983).

10 T. T. Wu and C. N. Yang, Nucl. Phys. B107, 365 (1976).

11 T. T. Wu and C. N. Yang, Phys. Rev. D 16, 1018 (1977).

12 A. R. Edmonds, "Angular Momentum in Quantum Mechanics", 2ed, Princeton Press, Princeton, N.J., 1974.

13 R. Morf, N. d'Ambrumenil, and B. I. Halperin, Phys. Rev. B 34, 3037 (1986).

14 B. I. Halperin, P. A. Lee, and N. Read, Phys. Rev. B 47, 7312 (1993).

15 G. Möller, S. H. Simon, to be published
16 We note in passing, that one could certainly construct several other trial wavefunctions which project in slightly different ways. For example, for $m>1$ we might consider $\tilde{\psi}_{i}\left(\vec{r}_{j}\right)=\mathcal{P}\left\{\psi_{i}\left(\vec{r}_{j}\right) J_{j}^{m_{1}}\right\} J_{j}^{m_{2}}$ with $m_{1}+m_{2}=m$ (and $\left.m_{1} \geq 1\right)$. In the cases we have checked we have found that wavefunctions built with this version of $\tilde{\psi}$ are also extremely similar to those built from Eq. 6 .

17 A subtlety in this process results from the observation that the wavefunction, calculated as described in the appendix A by separating it into a Slater-determinant of the projected pseudo single-particle-wavefunctions and a Jastrowfactor, tends to produce numerical instabilities in the determinant algorithm. This is easily understood, since two particles approaching each other at a distance $d$ will cause the respective $\tilde{\Psi}_{i}$ to grow as $\tilde{\Psi}_{i} \propto d^{-(|q|+n)}$ in the same manner, producing two linearly dependant columns in the matrix. Now, due to numerical errors, instead of obtaining zero when two columns become the same, the evaluation yields numerical errors following approximately the same power law behavior, that will dominate the result. Since the short distance behavior of the wavefunction is known, extrapolation allows us to correct for such events that occur with a probability of less than $10^{-4}$ in all of the presented calculations.

18 Note that Pan et al. ${ }^{8}$ use a a different normalization for $m^{*}$, which amounts to a slight rescaling as follows: $m_{\text {Pan }}^{*}=$ $\sqrt{B_{\nu} / B_{1 / 4}} m_{\text {nor }}^{*}$.

19 in this appendix, we note the number of flux pairs attached to each $\mathrm{CF}$ as $p$, according to Kamilla's notation, whereas $m$, that had been used for this purpose in section II to comply with the common notation for the filling fractions of Jain's series, is already used for the eigenvalue of $L_{z}$ here. 